work in a historical framework.

The second part of the book deals with history and includes biographical memoirs of several of his contemporaries, notably Landau, Tamm and Evgeni Lifshitz, but also Sergei Ivanovich Vavilov (brother of the geneticist Nikolai Vavilov and former president of the Soviet Academy of Sciences), as well as an autobiography. There is also an analysis of Russia's relationship with the Nobel prize, particularly in connection with C. V. Raman's prize for Raman scattering and the parallel work of G. S. Landsberg and L. I. Mandelstam, and with the discovery of the VavilovCherenkov effect. Finally, there is a collection of Ginzburg's publications on social and political questions, written with great factual detail and strong feeling.

In short, Ginzburg presents himself and his work, for the record, in the wider world of physics and of Russia.

Alan L. Mackay is in the School of Crystallography, Birkbeck College, University of London, Malet Street, London WCIE 7HX, UK.

\title{
Families behaving badly
}

\section{A Natural History of Families by Scott Forbes \\ Princeton University Press: 2005.248 pp. $\$ 27.95, £ 17.95$}

\section{Jonathan Wright}

I always feel that I am in a better position than many scientists when a friend or family member asks what my research is actually about. Compared with a theoretical physicist, it is relatively easy for me, as a behavioural ecologist, to enlighten and entertain members of the public, using the obvious parallels that exist between birds and humans in their family relationships. For example, there are disagreements between members of a pair over who does most of the childcare, and constant battles between overworked parents and their demanding offspring concerning apparently unimportant items of food. In his new book A Natural History of Families, Scott Forbes takes up this theme with a vengeance to reveal the many fascinating aspects of human family life and reproduction that arise from evolutionary conflicts of interest.

This 'popular' science book sells itself on the salacious revelation that all is not as nice as it seems within our cosy families and in the wonderful creation of an infant. As an avian biologist, the author presents a dispassionate account of parent-offspring conflict, siblicide and infanticide in animals and plants. But in the background lurks the disturbing fact that we are talking here about mothers and fathers, sons and daughters, and the family - an institution at the heart of human social experience.

The full horror is revealed when the author exposes at length the human side of the story. He describes research showing that 'morning sickness' and even pre-eclampsia (dangerously high blood pressure) during human pregnancy may be the manifestation of an evolutionary struggle between mother and child over placental blood flow and the frequent abortion of 'unfit' embryos. Fathers also get involved, and often on the side of the offspring. Certain genes become 'imprinted' only when inherited from the father, and these prompt the embryo to sequester even higher

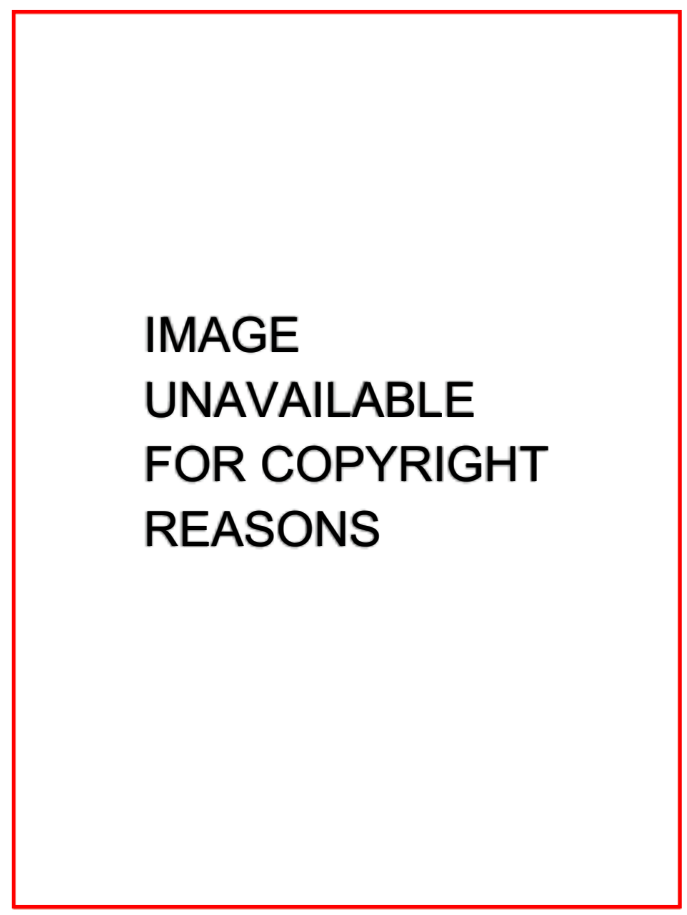

Who said we are meant to live in harmony? Makolm in the Middle sees the funny side of life in a dysfunctional family.

the ethical and philosophical implications of our own biology is not taken. Towards the end of the book, however, Forbes hints at a moral landscape. Somewhat negative language is used when describing current medical practices in assisted human reproduction. For example, the artificial implantation of multiple embryos increases the chance of a successful pregnancy, but inevitably it also increases multiple births of twins and triplets. This in turn creates its own problems (some as yet unknown) for the mother and especially for the future health and mental functioning of children born from artificial multiple pregnancies. The tragedy is that individual couples cannot afford the possibly healthier alternative of having fewer embryos implanted each time (because of the many more cycles that this would require), and most governments do not consider it their financial responsibility to step in, especially where older couples are concerned.

The analogy of Aldous Huxley's Brave New World is wheeled out to provide the usual warnings against the application of modern technology to human reproduction. Yet, evolutionarily speaking, Huxley's was perhaps a group-selectionist vision of excessive state control. The current 'customer-centred' health care that arises from free-market economics, and generates the social dilemma outlined above, might rather be described as an outcome of individual-level selection. But what, if any, should society's role be in moderating individual reproductive choices? I can't help feeling that the author, having implicitly introduced moral statements concerning modern human reproduction, bears some responsibility to discuss the ethical issues that follow from an evolutionary perspective if only to expose the complexity of the problem.

levels of resources from the mother.

Siblings are no better to one another, given the disturbing fact that some of us must have been murderers while in the womb: in more than $90 \%$ of human twin conceptions, mortality of one of the two embryos occurs. If we were a species of bird, this fact would classify us as an 'obligate brood reducer', along with those famously bloodthirsty little black eagle chicks.

How, then, do we react when we are told that these awful things are going on in our own families and inside our own bodies? More importantly, what do we think about the social and medical policies that perpetuate, or seek to modify, these phenomena? Does evolution provide a rationale for their continued existence, or a starting point for a better-informed programme of cultural and political change?

Unfortunately, the opportunity to explore
At the end of the day, this forayinto popular science perhaps does not do justice to the high quality of the author's own research on avian family conflict. In terms of outlining the underlying theory and non-human evidence, other texts, such as Douglas W. Mock's More Than Kin and Less Than Kind (Belknap Press, 2004), probably represent a better compromise between the popular and formal science formats. However, these do not attempt to deal explicitly with the tricky issues of human families and reproduction. In contrast, Forbes has taken on one of the hardest tasks in popularizing science, that of explaining to readers complex scientific findings about their own life and culture - and inevitably we all have our own opinions about that.

Jonathan Wright is in the Institute of Biology,

Norwegian University for Science and

Technology, Trondheim 7491, Norway. 